\title{
Status of the NICA Project
}

\author{
A.Sorin ${ }^{1}$ \\ Joint Institute for Nuclear Research, 141980 Dubna, Russia \\ E-mail: sorinetheor.jinr.ru
}

\section{V.Kekelidze}

Joint Institute for Nuclear Research, 141980 Dubna, Russia

E-mail: vladimir.kekelidzedcern.ch

\section{A.Kovalenko}

Joint Institute for Nuclear Research, 141980 Dubna, Russia

E-mail: kovalen@dubna.ru

\section{R.Lednicky}

Joint Institute for Nuclear Research, 141980 Dubna, Russia

E-mail: lednicky@fzu.cz

\section{V.Matveev}

Joint Institute for Nuclear Research, 141980 Dubna, Russia

E-mail: matveeveinr.ac.ru

\section{I.Meshkov}

Joint Institute for Nuclear Research, 141980 Dubna, Russia

E-mail: meshkovajinr.ru

\section{G.Trubnikov}

Joint Institute for Nuclear Research, 141980 Dubna, Russia

E-mail: trubnikovejinr.ru

The NICA (Nuclotron-based Ion Collider fAcility) project is now under active realization at the Joint Institute for Nuclear Research (JINR, Dubna). The main goal of the project is a study of hot and dense strongly interacting matter in heavy ion (up to $\mathrm{Au}$ ) collisions at the centre-of-mass energies up to $11 \mathrm{GeV}$ per nucleon. Two modes of operation are foreseen, collider mode and extracted beams, with two detectors: MPD and BM@N. The both experiments are in preparation stage. An average luminosity in the collider mode is expected as $1 \cdot 10^{27} \mathrm{~cm}^{-2} \mathrm{~s}^{-1}$ for $\mathrm{Au}(79+)$ collisions at ${\sqrt{\mathrm{s}_{\mathrm{NN}}}}=9 \mathrm{GeV}$. Extracted beams of various nucleus species with maximum momenta of $13 \mathrm{GeV} / \mathrm{c}$ (for protons) will be available. A study of spin physics with extracted and colliding beams of polarized deuterons and protons at the energies up to $27 \mathrm{GeV}$ and luminosity $1 \cdot 10^{32} \mathrm{~cm}^{-2} \mathrm{~s}^{-1}$ of (for protons) is foreseen with the NICA facility at the further stage. The proposed program allows one to search for possible signs of phase transitions and critical phenomena as well as to shed light on the problem of the nucleon spin structure.

9th International Workshop on Critical Point and Onset of Deconfinement

17-21 November, 2014

ZiF (Center of Interdisciplinary Research), University of Bielefeld, Germany

${ }^{1}$ Speaker 


\section{Introduction}

The new research facility NICA aimed at study of heavy ion and polarized proton and deuteron collisions has been under design and construction at the Joint Institute for Nuclear Research (JINR) since 2010. The facility and basic physics motivation of NICA construction were presented elsewhere, including the PoS edition, earlier [1,2]. The study of hot and dense baryonic matter should shed light on: in-medium properties of hadrons and the nuclear matter equation of state (EOS); the onset of deconfinement (OD) and/or chiral symmetry restoration (CSR); phase transition (PT), mixed phase and the critical end-point (CEP); possible local parity violation in strong interactions (LPV) [3]. It is indicated in a series of theoretical works, in particular, in [4] that heavy ion collisions at $\sqrt{S}_{\mathrm{SNN}}=4-11 \mathrm{GeV}$ allow attaining the highest baryon density. These calculations are illustrated in Fig. 1. The NICA research domain is attractive as being the expected region for searching of new phenomena at the maximum baryon density including possible phase transitions.

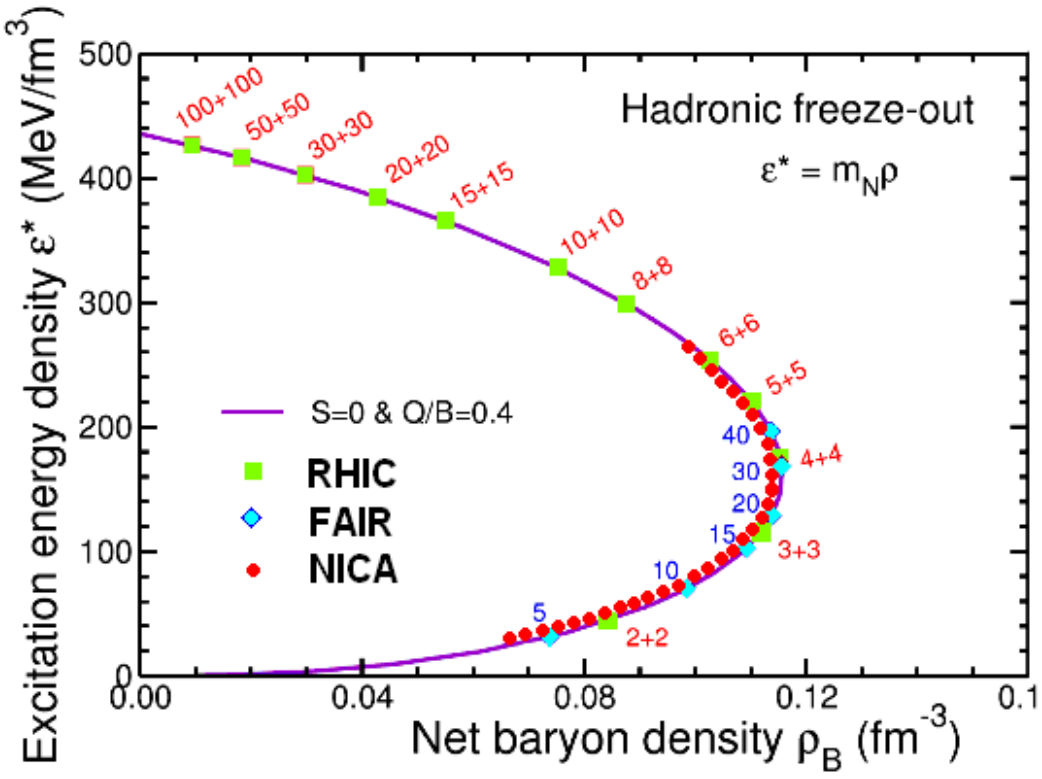

Figure 1: Freeze-out diagram for baryonic matter showing baryon density reachable at different facilities both in collider and fixed target experiments [4].

The study of the nucleon spin origin ("spin puzzle") and polarization phenomena in light and heavy ion interactions is another target of research at NICA. The expected high intensity and high polarization $(>50 \%)$ of colliding proton and deuteron beams could provide unique possibilities for these study.

The NICA first priority construction plan up to 2020 foresees an essential development of the existing accelerator facility and construction of the new spectrometer - Baryonic Matter at Nuclotron (BM@N) in order to start as soon as possible the fixed target experiments with heavy ion beams extracted from the modernized Nuclotron and the MultiPurpose Detector (MPD) at the NICA colliding beams with a primary goal of the obtaining new experimental data from heavy ion collisions. Thus the BM@M and MPD setups both will cover the collision energy range $\sqrt{ }_{\mathrm{S}_{\mathrm{NN}}}$ from 2 up to $11 \mathrm{GeV}$ for heavy ions and to about $13.6 \mathrm{GeV}$ for the light weight ones (up to sulphur or argon). 


\section{The accelerator facility development}

The Nuclotron facility is under operation at Veksler and Baldin Laboratory of High Energy Physics (VBLHEP) of JINR since 1993 [5]. The facility development in accordance with the NICA project includes: the extension of injection complex, design and construction of heavy ion booster, and the collider rings with two interaction points IP1 and IP2 aimed at the MPD and SPD detectors respectively (Fig. 2). The injection complex should provide a wide set of ion species up to the heaviest one. Au, at an energy of $3.5 \mathrm{MeV} / \mathrm{u}$ with intensity of $2 \cdot 10^{9}$ particles per cycle. The main efforts at the present time are devoted to completion of beam tests of both heavy ion, KRION-6T, and polarized proton and deuteron, SPI, sources aimed at reaching of the specified parameters and to completion of manufacturing of the HILAC and the new RFQ fore-injector that should replace the old high voltage pulsed transformer at the linac LU-20. The design and manufacturing of this new equipment is carried out in collaboration with German and Russian research laboratories and companies [6]. The RFQ of HILAC is under adjustment at Bevatech (Frankfurt). The completion of the injector's manufacturing and its tests at the accelerator together with the ion sources are expected by fall 2015 . The facility contains also the source of polarized ions (SPI) with the linac accelerating light ions up to $5 \mathrm{MeV} / \mathrm{u}$ that provides direct injection of polarized deuterons and protons (proton beam energy is $20 \mathrm{MeV}$ ) into the Nuclotron and then to the collider. The booster synchrotron should accelerate heavy ions up to $600 \mathrm{MeV} / \mathrm{u}$. The magnetic ring of $211 \mathrm{~m}$ long is planned to be placed inside the window of the Synchrophasotron yoke. Technical design of the booster systems was performed. The most specific features of the booster are ultrahigh vacuum and the electron cooling system.

he upgraded Nuclotron should provide proton, deuteron (including polarized) and multi charged ion beams with the maximum energies: $5.8 \mathrm{GeV} / \mathrm{u}$ for $(\mathrm{A}=2, \mathrm{Z}=1) ; 3.3 \mathrm{GeV} / \mathrm{u}$ for $\mathrm{Xe}$ $(\mathrm{A}=124, \mathrm{Z}=42)$; and $4.5 \mathrm{GeV} / \mathrm{u}$ for $\mathrm{Au}(\mathrm{A}=197, \mathrm{Z}=79)$. The ions are fully stripped before the injection into the Nuclotron.

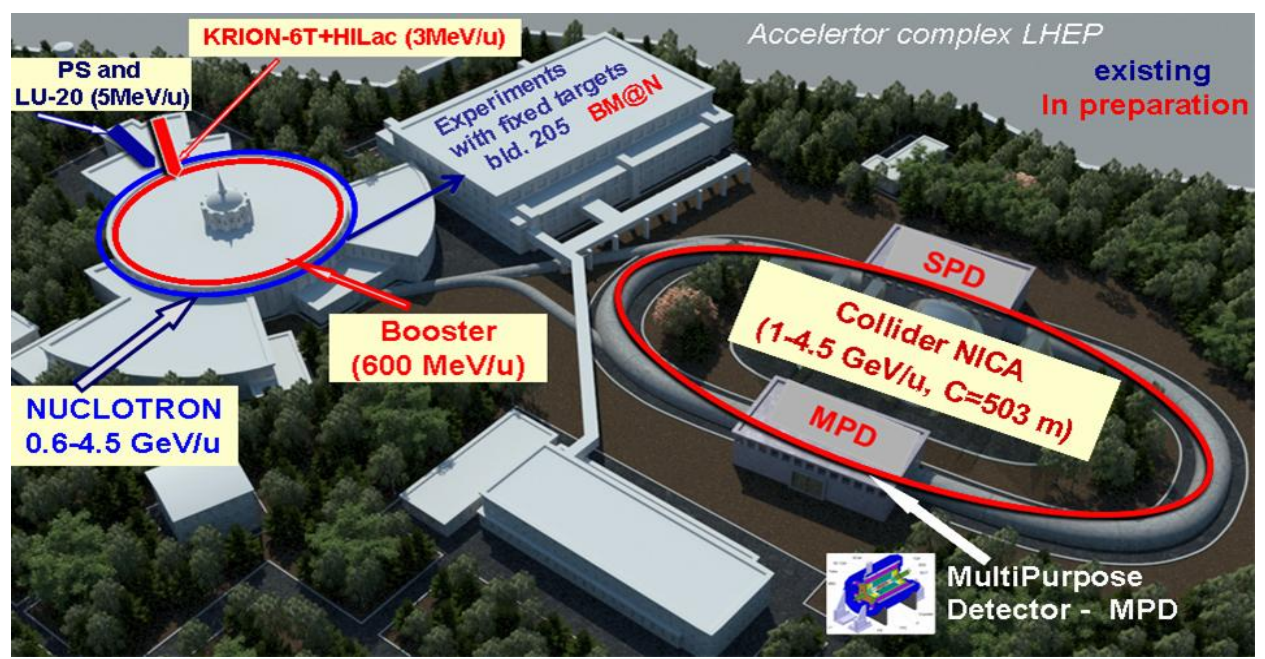

Figure 2: The NICA facility at LHEP JINR.

The two storage rings with two interaction points (IP). The major parameters of the NICA collider are the following: $\mathrm{B} \rho=45 \mathrm{Tm}$; vacuum in a beam chamber $-10^{-11} \mathrm{Torr}$; maximum dipole field $2 \mathrm{~T}$; ion kinetic energy range from $1 \mathrm{GeV} / \mathrm{u}$ to $4.5 \mathrm{GeV} / \mathrm{u}$ for Au79+; zero beam crossing angle at IP; $9 \mathrm{~m}$ space for detector allocations at IP's; average luminosity $\mathrm{L}=1 \cdot 10^{27}$ $\mathrm{cm}^{-2} \mathrm{~s}^{-1}$ for gold ion collisions at ${\sqrt{\mathrm{S}_{\mathrm{NN}}}}=9 \mathrm{GeV}$. The collider ring $503.04 \mathrm{~m}$ long (twice as large 
as the Nuclotron ring) has a racetrack shape and is based on double-aperture (top-to-bottom) superferric magnets - dipoles and quadrupoles. A superconducting NbTi composite hollow-tube cable for the magnets is designed and manufactured at the Laboratory. The first set of full size prototype magnets has been produced and passed all necessary tests. The serial fabrication of the magnets for NICA facility will be started at the SC magnet workshop at LHEP in 2016.

The NICA cryogenics is also under upgrade aimed at the increasing refrigerator power, making the new distribution system of liquid helium and other purposes. More details on the accelerator complex development are presented in [7].

\section{The heavy ion facilities}

The NICA place in the landscape of existing and future accelerators is illustrated in Figure 3 [1]. As one could conclude not only the future facilities but the existing ones are targeting at the energy region allowing exploration of baryonic matter at the maximum density.

The NICA facility at JINR will cover the relevant energy range in heavy ion collisions.

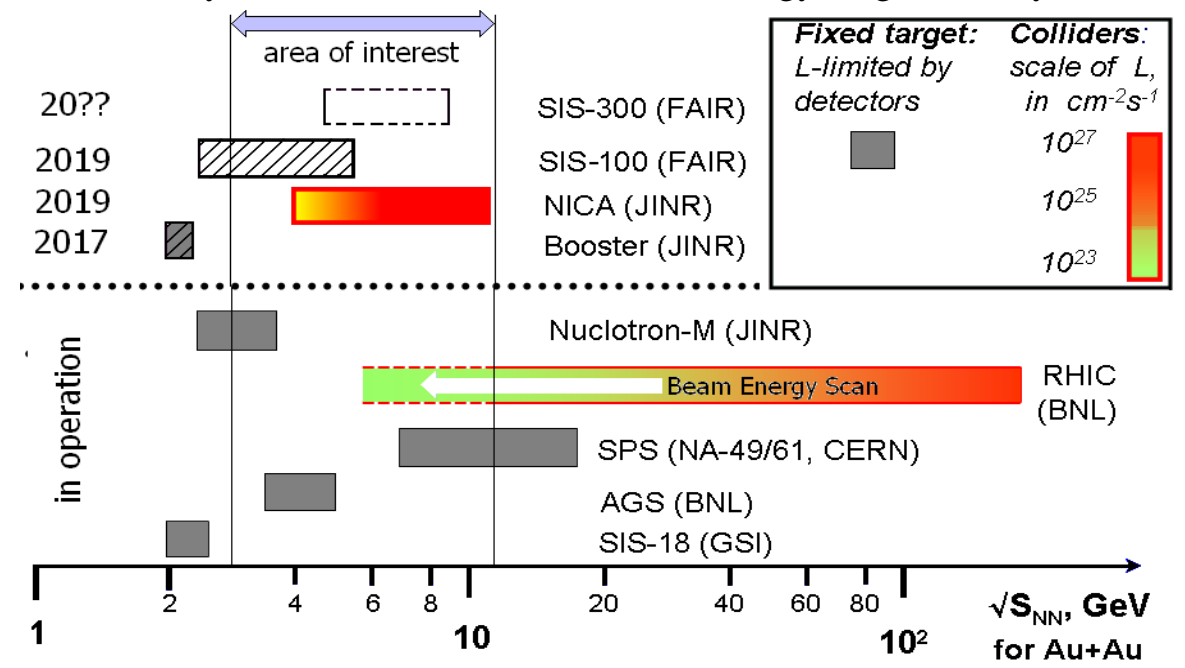

Figure 3: The landscape of present and future HI accelerators.

\section{The BM@N experiment}

1. The goals of research at the BM@N setup (Baryonic Matter at Nuclotron) are the following:

2. Heavy-ion collision A+A - study of the properties of dense nuclear (dominantly baryonic) matter with strangeness:

- production mechanisms and modifications of hadron properties in dense nuclear matter ("in-medium effects") using the following probes: strange mesons, strange and multi-strange baryons; vector mesons via hadronic or dilepton/photon mode).

- $\quad$ study of the EoS with strangeness;

- hyper-matter production: search for light hypernuclei and multi-strange metastable objects.

3. Study of elementary reactions: pp, pn(d) as "reference" to pin down nuclear effects.

4. Search for , cold" nuclear matter with $\mathrm{pA}$ - collisions.

BM@N at the first stage can study the in-medium effects on strangeness measuring of a variety of observables for different energies and centralities in heavy-ion collisions in order to find an "anomalous" behaviour in comparison with theory. The observables sensitive to in- 
medium effects are the following: particle yields and ratios pT- to (mT) -spectra, rapidity distributions, angular distributions, collective flow. The measurement of in-medium effects for vector mesons $(\mathrm{V}=\rho, \omega, \varphi)$ could be possible in perspective. An optimal way for that is studying of the $\mathrm{V} \rightarrow \mathrm{e}^{+} \mathrm{e}^{-}$mode or photon mode: $\omega \rightarrow \gamma \pi^{0}$. Possible alternative is $\varphi \rightarrow \mathrm{K}^{+} \mathrm{K}^{-}$ strong decay.

The BM@N setup (Fig. 4) includes: the existing wide aperture dipole magnet (SP-41); a tracking system consisting of 12 planes of high resolution gem chambers (GEM) and 8 planes of drift chambers (DCH); a time-of-flight (TOF) system based on the RPC chambers and a fast counter detector providing trigger signal; a zero degree hadron calorimeter (ZDC) for the reaction plane definition and estimation of the impact parameter.
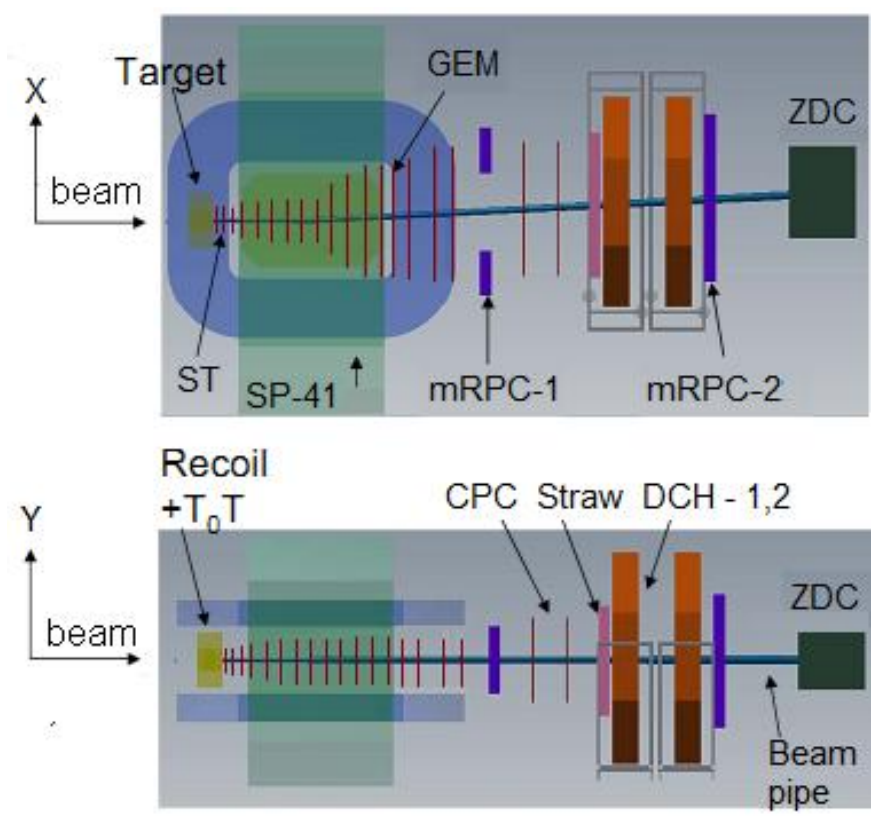

Figure 4: The BM@N setup: GEM - central tracker inside analysing magnet; DCH+Straw - outer tracker behind the magnet; mRPC+T0 - time-of-flight system; ZDC - centrality measurements.

The heavy-ion collision experiments at BM@N located in the fixed-target hall of the Nuclotron will provide competitive research program focused on physics of dense nuclear and strange matter. Substantial progress was achieved in the setup equipment manufacturing, its preparation and installation in the experimental hall during 2014. The first test run of the BM\&N basic elements is scheduled for February-March 2015 at the Nuclotron beam. The setup will be exposed to ion species from deuterons to xenon (optionally) over energy range from 5.2 to $1.3 \mathrm{GeV} / \mathrm{u}$ during 2016-2017 (before the booster will be put into operation).

The upgrade is foreseen at the second stage to equip the setup with a silicon vertex detector (in cooperation with GSI, Darmstadt), and with an electromagnetic calorimeter. The heavy-ion collision experiments at BM@N located in the fixed-target hall of the Nuclotron will provide competitive research program focused on physics of dense nuclear and strange matter.

\section{The MPD experiment}

The MPD experimental program is aimed to investigate both hot and dense baryonic matter and polarization phenomena. Preliminary list of the first priority physics tasks to be performed includes: 
1. measurement of a large variety of signals at systematically changing conditions of collision (energy, centrality, system size) using as bulk observables $4 \pi$ geometry particle yields (OD, EOS); multi-strange hyperon yields and spectra (OD, EOS); electromagnetic probes (CSR, OD); azimuthal charged-particle correlations (LPV); event-by-event fluctuation in hadron productions (CEP);

2. correlations involving $\pi, \mathrm{K}, \mathrm{p}, \Lambda(\mathrm{OD})$; directed and elliptic flows for identified hadron species(EOS,OD); reference data (pp-collisions) will be taken at the same 1 conditions;

3. study of hyperon polarization and other polarization effects after the setup upgrade.

The MPD is a typical collider detector based on a superconducting solenoid with the magnetic field of $0.5 \mathrm{~T}(\sim 5 \mathrm{~m}$ in diameter and $\sim 8 \mathrm{~m}$ in length). It will be installed in the IP-1 of the collider ring. The major sub-detectors of the MPD (Fig. 5) are: a time projection chamber (TPC); an inner tracker (IT) based on the silicon strip detectors; a time-of-flight (TOF) system based on the RPC modules; an electromagnetic calorimeter (ECal); end cap trackers (ECT). There are foreseen three stages of putting the MPD into operation. The first, start-up stage involves the magnet, TPC, TOF, ECal (partially), IT (partially). At the second stage the end caps of the MPD will be fully equipped and some readout systems modernized.
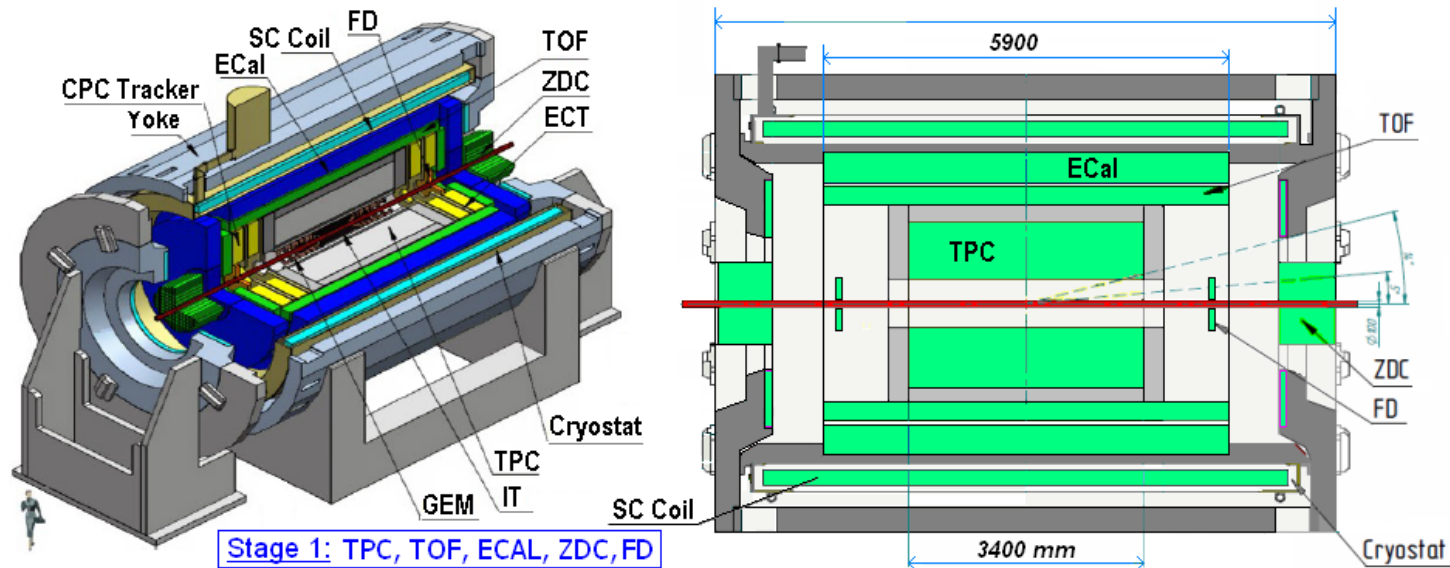

Figure 5: The MPD setup and the first stage elements (shown with green in the right scheme).

The processes studied with the MPD were simulated using the dedicated software framework (MpdRoot). This software is based on the object - oriented framework FairRoot [8] and provides a powerful tool for detector performance studies, development of algorithms for reconstruction and physics analysis of the data. The evaluated rate in $\mathrm{Au}+\mathrm{Au}$ collisions at the maximum energy (10\% central interactions) will be up to $7 \mathrm{kHz}$ taking into account the design luminosity of $\mathrm{L}=1 \cdot 10^{27} \mathrm{~cm}^{-2} \mathrm{~s}^{-1}$. Groups from 12 institutions are working on the sub-detector $R \& D$ and on prototyping all detector elements. More detailed information could be found in the conceptual design report [9]. It was shown that the MPD is well optimized for studying inmedium effects caused by high baryon densities, such as: changing particle properties in hot and dense medium (broadening spectral functions etc.), event-by-event dynamical fluctuations of strange to non-strange particle ratios and others. These studies could be done with better precision than ones performed at the current experiments. The simulation of MPD capabilities shows that high statistics of studied events could be accumulated $\left(10^{9}\right.$ minimum bias events and $10^{8}$ central events per week), which provides a precision femtoscopy study with respect to 
correlation of multi-strange particles. More than $10^{6}$ of $\Omega$-hyperon decays can be recorded in ten weeks running. The procedure of the identification and modeling results have been described in the previous publications, in particular in [1,2]. Special attention was devoted to the simulation of vector meson production and their di-lepton decays. The results have confirmed possibility of reliable identification of these reaction channels. There are many other proposals such as the studying vorticity [10], helicity separation in heavy ion collisions [11], $\phi(1020)$ - meson production [12] and others that could be studied at NICA. The best conditions are exists for observation and study light hypernuclei at both MPD and BM@N setups. The simulations (Fig.6) confirm enhanced yields of different single and double $\Lambda$ hydrogen and helium nuclei at the energy range covered by the NICA.

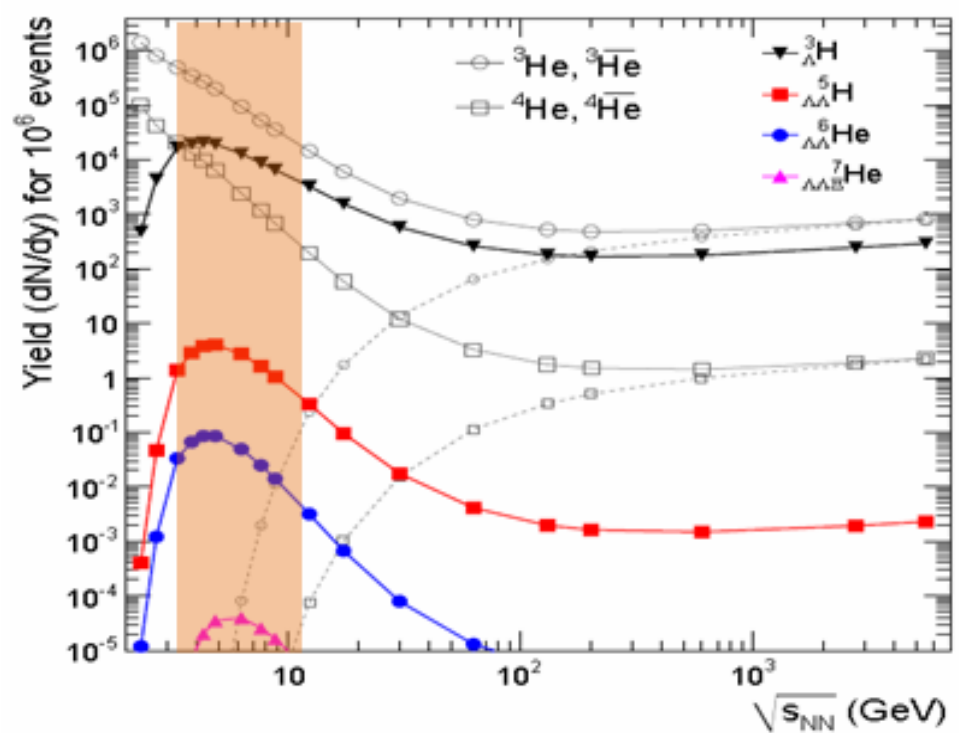

Figure 6: The yields of different single and double hydrogen and helium hypernuclei as function of centre- of- mass energy. The range covered by BM@N and MPD setups is marked with light brown.

The tests of the detector prototypes, which are necessary for the first stage of both BM@N and MPD setups, are carried out at the Nuclotron beams. Optimization of the MPD elements is practically completed. The technical design of the SC solenoid was also completed. Preparatory work to its manufacturing is in progress.

\section{SPD program}

The NICA program foresees that the SPD detector will be designed and installed in the IP2 to study spin physics. There is a number of processes which could be studied with this detecor and with the fixed target detectors at polarized beams extracted from the upgraded Nuclotron. These are in particular: MMT-DY processes with longitudinally and transversally polarized $\mathrm{p}$ and d beams; extraction of unknown (or poorly known) parton distribution functions (PDF); PDFs from $\mathrm{J} / \psi$ production processes; spin effects in various exclusive and inclusive reactions; cross sections of diffractive processes; helicity amplitudes and double spin asymmetries (Krisch effect) in elastic reactions; spectroscopy of quakonium with any available decay modes. This can be done in the kinematic energy region not available for other experiments. The analysis of the accelerator issues related to polarized proton and deuteron beam acceleration and the schemes of the polarization control in the Nuclotron and in the collider are presented in $[13,14]$. 


\section{Status of the construction}

The main R\&D work on the MPD components and the new accelerator components has been fulfilled by the end of 2014. The mass production of the SC - magnets and different MPD detector elements is planned to start in 2015. The preparation of the area for the collider and the detectors installation was started in 2013. The technical design project of the NICA collider building, the MPD and SPD halls and engineering infrastructure was completed in 2013. The project State expertise have been completed in 2014 also. At the current stage clarification of some specific question before approval of the contract with industrial company is occurred.

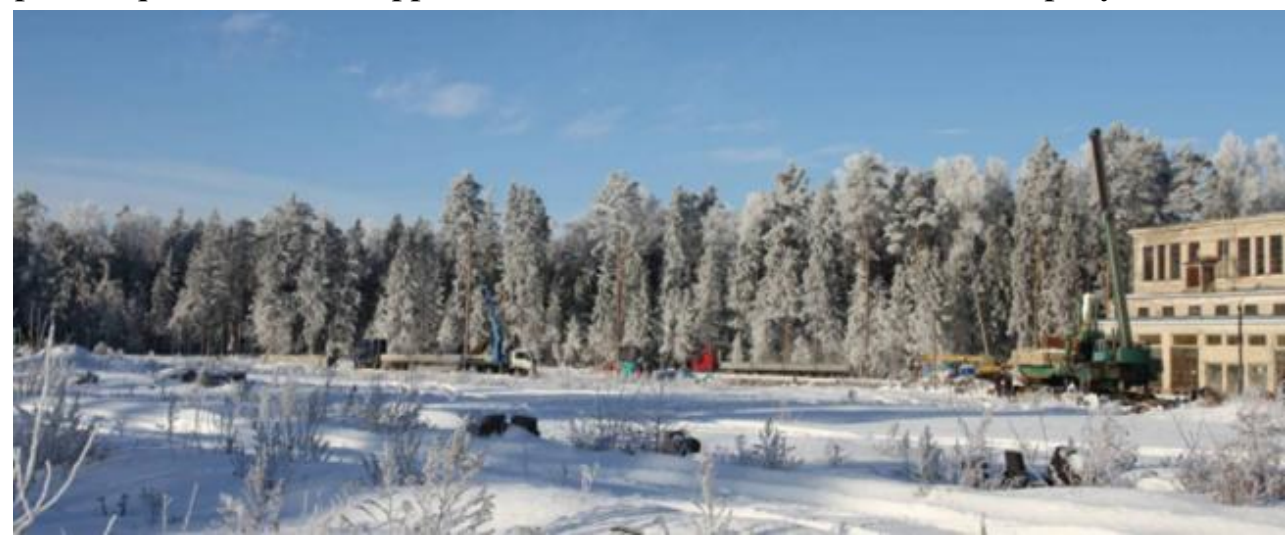

Figure 7. View of the NICA collider site at the Laboratory in December 2014.

The NICA accelerator and experimental complex and the corresponding research program will provide relevant research, which is both competitive and complementary to ones being carried out at other centers and facilities.

\section{References}

[1] V.D.Kekelidze et al., PoS (ICHEP2012) 411, (2013).

[2] V.D.Kekelidze et al., PoS (ISHEPP XXII) 002, (2014).

[3] A.Sissakian et al. Part.\& Nucl Lett. 5, 1 (2008).

[4] J. Cleymans, and J. Randrup, Phys. Rev. C74 (2006) 04791.

[5] A.D.Kovalenko. EPAC 94, Proceedings v. I, p. 161-164, (1995).

[6] A.Butenko et all. IPAC 2014, paper WEPRO067, Dresden, June 2014.

[7] G.Trubnikov et al. ibid, paper TUPRO005, June 2014.

[8] D.Bertini et al., JoP,CS,119, 032011, (2008). http://cbmroot.gsi.de.

[9] MPD CDR v.1.2 (JINR, Dubna, 2010); http://www.nica.ru.

[10] O.Rogachevsky et al, PR, C, v.82, (2010) 054910.

[11] M.Baznat et al., PR, C, v.88, (2013) 061901.

[12] L. Yordanova. PoS (ISHEPP XXII), 135, (2015).

[13] I.A.Savin et al. arXiv: 1408.3959[hep-ex], August (2014).

[14] A.D.Kovalenko et al, Part.\&Nucl Lett. Vol.45, Issue 1 (2014). 